\title{
Interplay between 5-HT and BDNF system in recombinant mouse strain upon chronic fluoxetine administration
}

\author{
Rodnyy A.Ya. \\ Institute of Cytology and Genetics \\ Novosibirsk, Russia \\ aleksandr1994rodny@gmail.com \\ Ilchibaeva T.V. \\ Institute of Cytology and Genetics \\ Novosibirsk, Russia \\ rbicehok@mail.ru
}

\author{
Kondaurova E.M. \\ Institute of Cytology and Genetics \\ Novosibirsk, Russia \\ kond_em@bionet.nsc.ru \\ Tsybko A.S. \\ Institute of Cytology and Genetics \\ Novosibirsk, Russia \\ antontsybko@bionet.nsc.ru
}

\author{
Antonov Y.V. \\ Institute of Cytology and Genetics \\ Novosibirsk, Russia \\ yegor@bionet.nsc.ru \\ Naumenko V.S. \\ Institute of Cytology and Genetics \\ Novosibirsk, Russia \\ naumenko2002@bionet.nsc.ru
}

\begin{abstract}
BDNF plays a key role in the development, differentiation, synaptogenesis and survival of brain neurons and in the processes of their adaptation to external impacts. Serotonergic (5-HT) system is another basic player in brain development and neuroplasticity. The study presents a comparative analysis of chronic fluoxetine treatment in recombinant mice differing in distal chromosome 13 fragment containing Htr1A gene of CBA mice strain on C57Bl6 genetic background. The problem here to be studied is mechanism of BDNF and 5-HT systems' interactions in antidepressant insensitivity. We measured mRNA and protein levels of BDNF, p75 ${ }^{\mathrm{NTR}}$, TrkB, 5-HT1A and 5-HT7 receptors, levels of 5-HT and its primary metabolite 5-Hydroxyindoleacetic acid (5-HIAA) in the brain structures that could have play primary role in mechanism of depression - frontal cortex and hippocampus. At the heart of the discussion are the different changes in BDNF system as well as in 5-HT system which allows us to conclude that the chronic fluoxetine injection increased depressive-like behavior in recombinant mice carrying distal chromosome 13 fragment containing Htr1A gene of CBA mice.
\end{abstract}

Keywords - fluoxetine; BDNF; 5-HT1A; 5-HT7; mice; serotonin

Depression appears to be the most widespread mental illness on the planet and represents one of the most urgent threats to humanity in the 21 st century. According to various sources, depression in the stage requiring active medical care affects $10 \%$ to $23 \%$ of the industrialized countries population, but despite a huge amount of studies, the mechanisms and pathogenesis of depressive disorders appeared to be still far from complete understanding. Disturbance in many neurotransmitter systems underlie the biochemical cause of depression. Among these a special place is occupied by the serotonergic, dopaminergic, cholinergic, GABAergic, norepinephrine systems as well as brain-derived neurotrophic factor system. Both BDNF and 5-HT systems play crucial role in mechanism of neurogenesis and neuroplasticity and are key players in mechanisms of depressive disorders.

Modern antidepressants block the serotonin transporter (5-HTT) suppressing the reuptake of serotonin from the synaptic cleft. However, from $20 \%$ to $40 \%$ of patients with depression are resistant to this class of antidepressants. Moreover, chronic antidepressant treatment is required to effectively correct depression. A key role in the regulation of the functional state of the brain serotonin system is assigned to the serotonin 5-HT1A receptor, which, through negative feedback, can inhibit the secretion of serotonin and the activity of serotonin neurons. Over the past 20 years, a lot of data has been accumulated confirming the participation of 5-HT1A receptors in the mechanisms of depression and in the action of antidepressants.
Modern neurobiology is actively studying the effect of genetic abnormalities on drug actions, because one or another genetic polymorphism can dramatically change the response to a drug. For example, changing the structure of receptors or transport molecules could essentially mediate signal transmission or drug delivery. It has been known that the same genes the different genetic backgrounds can differentially exhibit their functions, which leads to ambiguous reactions of drug therapy. Of great interest and broad prospects in the study of this problem are recombinant mice, which differ by the genome with the transferred or modified DNA fragment.

Recombinant B6.CBA-D13Mit76C (B6-76C) mouse line carrying distal chromosome 13 fragment containing HtrlA gene of CBA on the $\mathrm{C} 57 \mathrm{Bl} / 6$ genetic background is characterized by increase in postsynaptic and decrease in presynaptic functional responses mediated by 5-HT1A receptor in comparison with control B6.CBA-D13Mit76B (B6-76B) mouse line. The B6-76C showed decreased metabolism of 5-HT and increased BDNF/proBDNF ratio and these effects are accompanied by increased 5-HT1A receptor gene expression in hippocampus compared with B6-M76B mice. In addition to this after chronic fluoxetine treatment $\mathrm{B} 6-76 \mathrm{C}$ demonstrated decreased mobility in forced swim test which is commonly used to evaluate the effectiveness of antidepressants.

The aim of this study was to estimate the effect of chronic fluoxetine injection (20mg/kg, 14 days i.p.) on mRNA and protein levels of BDNF, proBDNF, p75 ${ }^{\mathrm{NTR}}$, TrkB, 5-HT1A receptors, its transcription factors FREUD1, FREUD-2 and 5-HT7 receptor, levels of 5-HT and its primary metabolite 5-HIAA in the frontal cortex and hippocampus of B6-76B and B6-76C mouse lines by using Western-Blot analysis, real time PCR and HPLC. These structures assumed to play the main role in pathogenesis of the depressive disorders. The data were analyzed using twoway ANOVA followed by Fisher's LSD post-hoc comparison.

The mRNA level of 5-HT1A receptor gene was decreased in cortex and in hippocampus in the B6-76C mice but not in the B6-76B mice after fluoxetine administration. However, 5-HT1A receptor transcription factors FREUD-1 and FREUD-2 were not altered. Chronic fluoxetine treatment led to decrease in level of 5-HT7 receptor gene expression in the cortex of B6-76C and in the hippocampus of $\mathrm{B} 6-76 \mathrm{~B}$ mice. The mRNA level of BDNF gene was increased in $\mathrm{B} 6-76 \mathrm{~B}$ in the cortex and in $\mathrm{B} 6-76 \mathrm{C}$ in the hippocampus after fluoxetine treatment compared with control groups. No differences in $\mathrm{p} 75^{\mathrm{NTR}}$ and TrkB receptors genes expression in cortex and hippocampus of the both lines were determined. 
We have also found significant changes in serotonin and 5-HIAA upon chronic fluoxetine treatment. Decrease in serotonin and 5-HIAA levels in the cortex of both lines and in the hippocampus of B6-76B mice was shown. The 5HIAA/5HT ratio was not changed in all investigated brain structures in both mouse lines after chronic fluoxetine treatment.

Western-Blot analysis revealed intriguing changes in BDNF system. BDNF protein levels were significantly higher in B6-76C after chronic fluoxetine treatment in frontal cortex and hippocampus. BDNF precursor proBDNF protein level was lower in both lines after fluoxetine in frontal cortex. There no proBDNF protein changes in hippocampus were found. Chronic fluoxetine treatment also failed to affect 5-HT1A, 5-HT7 receptors protein in investigated brain structures and TrkB-FL, TrkB-T1 and p75 ${ }^{\mathrm{NTR}}$ receptors protein in hippocampus. However, in frontal cortex TrkB-T1 was lower in B6-76B after fluoxetine treatment.

Thus, classic antidepressant increased depressive-like behavior which is accompanied by decreased the expression of post-synaptic 5-HT1A receptor, increased expression of $\mathrm{BDNF}$ genes and its product BDNF protein in hippocampus of B6-76C mice and decreased proBDNF level in B6-76C after fluoxetine treatment. It was found that changes in sensitivity of the 5-HT1A receptors results in the significant changes in the response to chronic antidepressant treatment.

\section{ACKNOWLEDGMENT}

The work was supported by Russian Scientific Foundation, project No.19-15-00027. 